\title{
MATEMÁTICAS EN EL PERÚ: Un caso de responsabilidad social ${ }^{*}$
}

\author{
Norma Arias \\ MAGÍSTER EN ADMINISTRACIÓN DE ESAN (PERÚ) \\ ariasn@esan.org.pe
}

\section{Resumen}

Un grupo empresarial de reconocida trayectoria constata que existe una relación muy importante entre desarrollo y nivel educativo de la población y decide contribuir a mejorar la enseñanza de las matemáticas como un medio para elevar el rendimiento escolar. Empieza desarrollando textos escolares con una metodología novedosa y luego, con auspicio de otras empresas, consigue donarlos a centros educativos públicos de diferentes lugares del país. Poco después, el programa, denominado Matemáticas para Todos, busca incrementar su cobertura y da el salto al formato virtual, con una propuesta interactiva que apela al juego y a la diversión para captar la atención del niño y del adolescente.

Palabras clave: educación, matemáticas, método interactivo, software educativo, responsabilidad social, asociación civil, Perú, estudio de caso.

\begin{abstract}
A renowned corporate group states that there is a very important relationship between the development and the educational level of a people, and so decides to improve the mathematics school program as a way to improve academic performance. It starts by developing school texts with a new methodology and then, sponsored by other companies, it donates them to public educational institutions around the country. Shortly after, the program, Math for Everyone, seeks to increase its coverage and leaps to a virtual format, with an interactive proposal geared towards fun and games in order to catch the child and teenager's attention.
\end{abstract}

Key words: education, mathematics, interactive method, educational software, social responsibility, civil association, Peru, case study.

C omo se muestra en este caso de es tudio, la responsabilidad social de la empresa es una decisión voluntaria, no impuesta. Cada organización tiene una filosofía que se fundamenta en los valores de los ejecutivos y los empresarios que trabajan en ella.
Lo más importante de la responsabilidad social empresarial es el compromiso real que las empresas asumen con la sociedad, siendo consecuentes con el medio que les ha permitido realizar sus actividades económicas. De este modo generan un mayor beneficio para la

* Una versión anterior de este caso fue premiada en el Primer Concurso de Casos sobre Responsabilidad Social organizado por la Universidad ESAN, con auspicio de la Asociación Atocongo, en el primer semestre de este año (2005). 
colectividad y contribuyen a mejorar la calidad de vida de la población.

\section{La empresa: el Grupo Apoyo}

El Grupo Apoyo fue fundado por Felipe Ortiz de Zevallos en 1977. En más de 25 años de existencia y gracias a su permanente vocación por la excelencia, ha logrado una posición de liderazgo y reconocimiento en los diversos servicios que presta. En todo este periodo, sus ingresos han crecido a una tasa anual promedio de $25 \%$.

La creciente globalización de la economía implica que las fronteras nacionales se diluyan para la actividad empresaria. Por ello, desde 1997 el grupo ha iniciado una etapa de expansión regional estratégica, pues considera que cada vez más clientes que comercien o inviertan en el Perú y en otros países andinos provendrán de naciones vecinas.

Actualmente el grupo cuenta con 250 trabajadores y está integrado por diez organizaciones, como se puede observar en el siguiente cuadro.
La declaratoria de misión, visión y principios del grupo (www.apoyo.com) podrá dar una idea general de los objetivos que persigue como organización, de su preocupación por contribuir no sólo a la competitividad de sus clientes, sino también al crecimiento económico del país y el respeto a la democracia, así como por la carrera de sus trabajadores.

\section{Misión}

Esclarecer el proceso de toma de decisiones operativas y estratégicas de sus clientes a través de información precisa, objetiva y pertinente; de conocimientos actualizados; y de sabiduría sobre los medios en que actúa.

\section{Visión}

Constituirse como un faro externo valioso de información y conocimiento para los ejecutivos líderes de aquellas organizaciones que aspiran a lograr una competitividad global.

\section{Principios}

Los principios que rigen el diario quehacer del grupo son los siguientes:

\section{Estructura del Grupo Apoyo}

\begin{tabular}{lll}
\hline $\begin{array}{c}\text { Institución sin } \\
\text { fines de lucro }\end{array}$ & Filiales principales & \multicolumn{1}{c}{ Otras empresas } \\
\hline \multirow{2}{*}{ Instituyo Apoyo } & Apoyo Consultoría & Apoyo Administrativo \\
& Apoyo Opinión y Mercado & AC Inmobiliaria (Acisa) \\
& Apoyo Comunicación Corporativa & AC Capitales SAFI \\
& Apoyo Publicaciones & $\begin{array}{l}\text { Apoyo Opinión y Mercado Bolivia } \\
\text { Consultor Apoyo Ecuador }\end{array}$ \\
\hline
\end{tabular}

Fuente: www.apoyo.com. 
1.La vocación por la verdad

Considera fundamental difundir información veraz, precisa, oportuna y valiosa, así como procurar un análisis objetivo e inteligente de los hechos.

2.El compromiso con la sociedad Aspira a la promoción del crecimiento económico, el respeto a la Constitución y las leyes, la afirmación de la democracia política.

\section{La calidad del servicio}

Aspira a innovar continuamente para responder a los requerimientos del mercado y adecuar su organización a los desarrollos tecnológicos.

4.El desarrollo del personal

Aspira a ofrecer a cada uno de sus trabajadores una evaluación imparcial del desempeño, y capacitación y entrenamiento continuos.

5.La responsabilidad del trabajador Los trabajadores procuran hacer suyos los objetivos y políticas de la organización para asumir crecientes responsabilidades en la identificación de oportunidades y solución a problemas.

6.El espíritu de equipo

Se busca una comunicación abierta y sincera, sin dejar de reconocer que compartir experiencias contribuye a un mayor desarrollo intelectual y cultural.

7.La independencia y transparencia

La opinión editorial es autónoma e independiente de cualquier grupo de poder.

8.El crecimiento de la empresa

Busca el crecimiento y una rentabilidad atractiva sobre la base de la creatividad e innovación, un posicionamiento adecuado, el aprovechamiento de sus ventajas comparativas, la austeridad y la eficiencia en el uso de recursos disponibles.

\section{El Instituto Apoyo}

El Instituto Apoyo es una organización sin fines de lucro que nace en 1989 por iniciativa del presidente y los miembros del directorio del grupo del mismo nombre. $\mathrm{Su}$ misión es generar y difundir información y análisis para la promoción de un desarrollo económico sostenible, la ampliación y mejora en las opciones de políticas públicas, y el fortalecimiento de la democracia.

Actualmente contribuye al bienestar de la sociedad a través de la ejecución de proyectos educativos dirigidos a mejorar la enseñanza de la economía, las matemáticas y la gestión empresarial en el Perú.

En 1997 elaboró el texto: Economía para todos, con el objetivo de mejorar la enseñanza y comprensión de la economía en el ámbito escolar. Este libro resultó atractivo para los estudiantes porque presenta ejemplos y actividades que guardan relación con sus propias vidas e intereses.

Más adelante y en cooperación con un grupo de empresas privadas interesadas en la educación, Apoyo preparó una serie de informes sobre diversos sectores productivos del Perú: petróleo y gas natural, pesca, minería, electricidad, turismo y transporte, con el fin de que puedan distribuirse como suplementos de Economía para todos a los estudiantes secundarios de edades comprendidas entre 14 y 16 años.

El proyecto actual del Instituto Apoyo es Matemáticas para Todos, cuyo principal objetivo es la renovación de la enseñanza de esa materia para revertir la alarmante situación en que se encuentra su aprendizaje en el Perú. El proyecto se inició en el año 2000 con la elaboración 
de textos escolares, pero ahora se emplea las herramientas informáticas más avanzadas y se ha desarrollado una página web interactiva: www.20enmate.com. Con ello se busca dar a los niños mayor autonomía en el proceso de aprendizaje y motivarlos a ser perseverantes para conseguir los resultados que ellos quieran lograr a través de su esfuerzo.

El equipo de e-business del Grupo Apoyo sigue agregando valor a www.20enmate.com y a nuevos complementos virtuales. En un periodo de tres o cuatro años esperan demostrar que es perfectamente posible obtener buenos resultados en el aprendizaje de las matemáticas en colegios estatales, es decir, en alumnos de bajos recursos, si se elabora un programa y materiales adecuados y si se cuenta con la colaboración de los profesores y las autoridades.

El instituto planea desarrollar proyectos similares en otros temas y para otros públicos objetivo.

\section{El programa: Matemáticas para Todos}

Actualmente, el Perú ocupa el último lugar de Latinoamérica en rendimiento escolar en matemáticas. Según las estadísticas internacionales, hay una relación directa entre el desarrollo de los países y el rendimiento escolar: a mayor pobreza, menor rendimiento. La mayoría de escolares egresan del colegio sin haber adquirido habilidades básicas de cálculo mental, técnica operativa, razonamiento matemático ni geometría. Ello porque se obliga a los escolares a memorizar definiciones y a aplicar fórmulas mecánicamente, sin comprender lo que están haciendo; de modo que sólo se consigue aburrimiento y desmotivación. La metodología de enseñanza carece de una secuencia organizada y coherente.

\subsection{Diseño y evolución del proyecto}

Ante la situación descrita, el Instituto Apoyo diseñó y ejecutó el programa Matemáticas para Todos, que inicialmente comprendió la elaboración de textos escolares, con una metodología novedosa, y la donación de estos libros a unos 50 mil niños de colegios estatales ubicados en 21 departamentos del país. Para la donación se solicitó el auspicio de empresas privadas.

La metodología que promueve Matemáticas para Todos parte de la realidad e intereses de los niños a fin de estimularlos a participar en la solución de casos y problemas cotidianos. El objetivo es lograr que los niños de todos los sectores socioeconómicos desarrollen un razonamiento lógico-matemático que les permita entender, plantear y resolver problemas reales, así como descubrir el valor práctico de sus conocimientos, de modo que las matemáticas lleguen realmente a gustarles. Se trata de potenciar el desarrollo del niño y del adolescente con insumos adecuados que lo ayuden al tránsito exitoso del pensamiento concreto al pensamiento abstracto.

Entre los años 2001 y 2002, el Instituto Apoyo realizó una búsqueda internacional para identificar la metodología de enseñanza que mejor se ajustara a sus propósitos. Se interesó en la colección Lambacher Schweizer, de la editorial alemana Klett, especializada en la producción de textos escolares de matemáticas para 9 distintos niveles de estudio, y suscribió un convenio con esta editorial para la adap- 
tación de la colección a la realidad peruana. Los primeros resultados del convenio fueron los textos escolares Matemáticas para todos para primero y segundo de secundaria. En los años siguientes se publicó textos para el quinto y el sexto grado de primaria.

A fin de que el programa no se viera limitado por la situación de pobreza que afecta a la mayoría de escolares, en el año 2002 el Instituto Apoyo se planteó como meta lograr que las empresas privadas interesadas en mejorar la educación en el Perú donaran ejemplares de Matemáticas para todos a los estudiantes de primero y segundo de secundaria de las escuelas públicas administradas por Fe y Alegría en todo el ámbito nacional.

En abril del 2002 se inició la campaña de recolección de fondos y se consiguió que, como parte de sus acciones en favor de la comunidad, diversas empresas privadas donaran más de 23 mil ejemplares de Matemáticas para todos a escolares de gran parte de departamentos del Perú. Entre agosto y diciembre de ese mismo año, el instituto empezó el proceso de inducción con visitas de campo a localidades y colegios beneficiarios de la campaña. De esta manera, en abril del 2003 se alcanzó a distribuir textos para primero y segundo de secundaria a 96 centros educativos de diversas zonas de Lima y del interior del país.

En enero del 2003, el Instituto Apoyo inaugura Interaprendizaje, propuesta de entrenamiento a docentes que enseñan con Matemáticas para todos. Interaprendizaje atiende a profesores de diferentes zonas del país en forma descentralizada, mediante los textos Matemáticas para todos y videos de capacitación a distancia.
Se logró una cobertura de aproximadamente 300 profesores de matemáticas de dichos colegios.

Los talleres de Interaprendizaje son administrados por los propios docentes, quienes trabajan con ayuda de clases demostrativas y todas las herramientas del programa: metodología, ruta de aprendizaje, textos escolares, material para desarrollo y página web.

\subsection{Participación de otras instituciones}

Diversas organizaciones públicas y privadas han respondido a la convocatoria del Instituto Apoyo, lo que ha hecho posible la ampliación de la cobertura del programa.

El Banco de Crédito del Perú apoya las acciones en el ámbito nacional; su aporte permitió atender a ocho mil escolares en el año 2003 y a seis mil escolares en el año 2004, así como la creación y funcionamiento de la página web www.20enmate.com, concebida como un instrumento para el autoaprendizaje de alumnos y profesores.

Por dos años consecutivos, algunas empresas privadas han dado su ayuda solidaria a colegios estatales -y en convenio con el Estado- en sus áreas de influencia, donando textos para la creación de bancos de libros. Entre estas se puede mencionar a: Compañía de Minas Buenaventura S.A.A., en Huancavelica; Cementos Pacasmayo S.A.A., en Pacasmayo y Rioja; San Fernando, en Chincha; Southern Perú, en Tacna, Moquegua e Ilo; y Repsol YPF, en Ventanilla.

En el año 2004 se incorporaron a esta estrategia zonal Cementos Lima S.A. y la 
Asociación Atocongo, en los distritos de Villa El Salvador, San Juan de Miraflores y Villa María del Triunfo; Minsur S.A., en Puno; La Positiva Seguros y Reaseguros, en Arequipa; Corporación S.A., en Arequipa y Pisco; Barrick Misquichilca, en Huaraz y Alto Chicama. Otras colaboradoras del programa son Quimpac S.A. y Montana.

Durante el año 2003 las empresas Gloria S.A., en Arequipa, y Plaza Vea, en diversos distritos de Lima, también fueron colaboradores de Matemáticas para Todos. Asimismo, lo fueron Cofide y Ferreyros.

Finalmente, con el objetivo de orientar y canalizar los aportes del sector privado para la educación escolar del país, el Ministerio de Educación y el Instituto Apoyo firmaron en setiembre del 2003 un convenio por tres años a fin de promover el uso del material educativo donado, lo que permitirá la realización de programas de capacitación y la implementación de bibliotecas escolares.

\subsection{Página web: 20enmate.com}

Como parte de la plataforma educativa de Matemáticas para Todos, orientada a mejorar la competitividad de los jóvenes peruanos, se consideró esencial el desarrollo de una página web que complementara los textos escolares. No sólo porque esta herramienta refuerza lo aprendido en clase y permite que el niño amplíe su conocimiento y mejore su metacognición, sino porque en la era de la información es necesario saber utilizar Internet como un insumo básico para alcanzar competitividad.

20enmate.com es una página web interactiva y de acceso directo para la ense- ñanza divertida de las matemáticas; se trata de un proyecto que involucra a personal de sistemas, expertos en educación, administradores de empresas, comunicadores, escolares, padres de familia, profesores, directores de colegios, medios de comunicación, empresas privadas, propietarios de cabinas de Internet y hasta capacitadores voluntarios.

Primera en su género en el Perú, es innovadora y su diseño recoge elementos gráficos que despiertan el interés de los niños y motivan la interacción con los retos propuestos. No se ha encontrado en el Perú, ni en la región, otra página virtual similar dirigida a escolares. Los estudios realzados han demostrado su gran capacidad para captar la atención de niños y jóvenes.

Gracias a las acciones de comunicación y difusión que se desarrollaron con el apoyo decidido del Banco de Crédito del Perú (concursos por Internet, promociones con cabinas, diversos talleres con alumnos y profesores, etc.), se logró que en un año más de 100 mil niños visitaran y utilizaran la página.

El proceso de desarrollo de la página se hizo a un ritmo acelerado y tomó aproximadamente cuatro meses, de enero a abril del 2003. Primero se investigó los sitios de matemáticas que había ya en Internet. Luego se elaboró un diseño preliminar que fue probado, a través de diversos focus group, en los colegios Fe y Alegría de San Martín de Porres y en el colegio Trener. Se quería observar cómo manejaban los niños la página y qué les llamaba la atención. También se hicieron entrevistas en profundidad a profesores y padres de familia. Con estos insumos se desarrolló un producto piloto que fue 
probado con los mismos niños. La página ya contaba con diversas secciones que permitían al usuario autoevaluar su rendimiento, aprender jugando, hacer consultas específicas al doctor Mate y conocer estadísticas diversas, funciones en las que se percibe la importancia y aplicación práctica de los números. Esta vez gustó mucho la interactividad de la página y su facilidad para la navegación. El producto estaba casi listo. Finalmente, en las diferentes dinámicas de investigación se creó el nombre: www.20enmate.com. La página está en constante revisión y ahora incluye también estadísticas sobre temas económicos, poblacionales, de deportes, música, aspectos todos que demuestran la importancia y la aplicación práctica de los números en la vida diaria.

Como parte de las actividades de 20enmate, el 1 de setiembre de 2003 se lanzó el primer concurso de matemáticas por Internet denominado Matemáticas para Todos, cuyo principal objetivo fue incentivar el uso de Internet entre los escolares, especialmente en el ámbito de la educación matemática en el Perú. El concurso cubrió el ámbito nacional y participaron alumnos de primero, segundo y tercero de secundaria, incluso de lugares tan alejados como Puno y Cajamarca.

\subsection{Medición de resultados}

El éxito del programa se determina por los siguientes resultados y sus correspondientes indicadores:

- Que los textos lleguen a las aulas y sean utilizados por los estudiantes. En este caso los indicadores son: actas de recepción, actas de compromiso, actas de matrícula de niños atendidos por el programa.
- Que los profesores se actualicen y apliquen los lineamientos pedagógicos propuestos. Los indicadores son las fichas de monitoreo y las visitas a los colegios.

- Que los libros retornen al banco de libros para que puedan ser utilizados por otros alumnos al año siguiente. La ficha de censo de libros es un verificador de este resultado.

- Que los alumnos logren avances en su conocimiento de las matemáticas. El instituto se mantiene en contacto permanente con los profesores para registrar sus percepciones y hallazgos con relación a los alumnos.

\subsection{Reconocimientos}

En el año 2003 la página web 20enmate.com se hizo acreedora a tres premios. El primero le fue otorgado por el concurso Creatividad Empresarial, en la categoría Comunicaciones; el segundo, por la revista IT Users, en la categoría Portales; y el tercero, por la revista $P C$ World, en la categoría Mejor Iniciativa Tecnológica con Proyección Social.

En el año 2004 la página obtuvo cuatro nuevos premios. El primero correspondió al concurso La Mejor Página Web Peruana, en la categoría Educación y Ciencia; se le premió por su creatividad, diseño, funcionalidad, navegabilidad, interactividad, programación, mecanismos para presentar contenidos y logro de objetivos. La revista IT Users le otorgó dos premios, uno en la categoría Software Educativo por la sección «Autoevalúate», y otro en la categoría Software de Mensajería por la sección «Dr. Mate». El cuarto premio, llamado Crystal Obelisk for Social Responsibility, le fue otorgado por 
The World Foundation WEPR, de Nueva York, en la categoría Mejor Programa de Responsabilidad Social de una Empresa Privada, con Presupuesto Menor a $100 \mathrm{mil}$ dólares, por estar entre los cinco mejores programas de responsabilidad social, junto a renombradas empresas e instituciones. La Fundación WEPR organiza desde 1990 el único certamen mundial en la industria de Relaciones Públicas dedicado a premiar a los programas más efectivos de responsabilidad social.

Entre el 21 y el 25 de julio de 2004, el Instituto Apoyo fue invitado a participar en dos conferencias de alcance mundial, ambas llevadas a cabo en Orlando, Florida: International Conference on Education and Information Systems, Technologies and Applications (EISTA 2004) e International Conference on Cybernetics and Information Technologies, Systems and Applications (CITSA 2004). En estos encuentros el programa se presentó como un caso de estudio (ver en anexo: Playing With Mathematics In Peru Through Internet: A Case Study Of www.20enmate.com) enfocado en las características del site, el soporte de sistemas y el diseño de la network (estrategia de difusión y comunicación, estrategia con cabinas, colegios, niños, profesores, empresa privada, voluntariado, etc.), factores críticos para la sostenibilidad del producto a lo largo del tiempo.

\section{Lecciones aprendidas}

El programa Matemáticas para Todos tiene un objetivo mucho más ambicioso que el promedio de proyectos de responsabilidad social, pues no está destinado sola- mente a resolver un problema de corto plazo que afecta el entorno inmediato de la empresa que lo promueve -para permitir así una convivencia más armoniosa con la comunidad-, sino que busca un cambio estructural en la educación nacional, cambio que en el mediano plazo tendrá un impacto generalizado en las habilidades matemáticas de los peruanos $\mathrm{y}$, por ende, favorecerá la competitividad del país.

El Grupo Apoyo quería focalizar mejor su contribución en el área de responsabilidad social, y el proyecto Matemáticas para Todos cristaliza muchos valores que están asociados a lo que el grupo hace: hard facts, precisión, análisis, entre otros. La filosofía que inspira a sus miembros demuestra un real interés por la igualdad de oportunidades, porque el proyecto favorece sobre todo a los sectores marginados.

En este punto cabe preguntarse si la responsabilidad social empresarial es viable para la empresa peruana. La responsabilidad social representa una inversión que la empresa debe realizar para incrementar su rendimiento financiero y mantenerse en el mercado. Los beneficios son tanto cualitativos como cuantitativos. Los programas de proyección social mejoran la imagen y el nivel de aceptación de la empresa por los consumidores; por lo tanto, incrementan las ventas.

Además, en este caso de estudio se puede comprobar que la responsabilidad social implica programas de desarrollo con vida propia en el largo plazo. En caso contrario, la responsabilidad social de la empresa habrá fracasado. 


\section{Referencias bibliográficas}

ARIAS PICÓN, Norma. 2003. La relación entre el comportamiento ético, responsabilidad social empresarial y los resultados del negocio en el Perú. Lima: ESAN. Tesis para optar el grado de magíster en Administración.

SCHWALB, María Matilde y GARCÍA, Emilio. Buenas prácticas peruanas de responsabilidad social empresaria-Colección 2004. Lima: Centro de Investigación de la Universidad del Pacífico. Diciembre 2004.

GRUPO APOYO. Nuestra responsabilidad social [www.apoyo.com/resp_social/], 5 de junio del 2005.
PERÚ 2021. Ética y responsabilidad social [www.peru2021.org/ peru2021/artículos/ EticayResponsabilidadSocial.pdf], 5 de junio del 2005.

DELGADO-APARICIO L., ÁLVARO; TORRES LLOSA, Cecilia y ORTIZ DE ZEVALLOS, Gabriel. 2004. Playing with Mathematics in Peru through Internet: A case study of www.20enmate.com. Grupo Apoyo. [www.20enmate.com/pdf/ EISTAcaso.pdf], junio del 2005. 


\title{
Anexo
}

\section{Playing with Mathematics in Peru through Internet: A case study of www.20enmate.com ${ }^{1}$}

\author{
Álvaro Delgado-Aparicio L. \\ MANAGer of THE Holding GROUP, GRUPO APOYO \\ adal@apoyo.org.pe \\ Cecilia Torres Llosa \\ Educational Projects Director, Instituto Apoyo \\ ct@iapoyo.org.pe \\ Gabriel Ortiz de Zevallos \\ EXECUTIVE DiRECTOR, Instituto APOYO \\ goz@iapoyo.org.pe
}

\begin{abstract}
www.20enmate.com is an interactive website designed to improve mathematical skills among Peruvian primary and secondary school students. The site seeks to strengthen the children's sense of motivation and self-sufficiency through an innovative didactic methodology that stresses the idea that learning and practicing mathematics can be both fun and rewarding. www.20enmate.com has achieved outstanding qualitative and quantitative preliminary indicators, a result of its creative design and well-coordinated network of students, teachers, schools, parents, local Internet booths, the private sector and the general community. The design and implementation of this website is an initiative of Grupo Apoyo and its social responsibility program.
\end{abstract}

Key words: mathematics, e-learning, sustainability, Mathematics for Everyone, interactive website, educational network.

\section{Introduction}

According to the $\mathrm{UNESCO}^{2}$, Peru occupies the last place in school mathematics in Latin America. In order to revert this situation, Grupo Apoyo has oriented its social responsibility program towards the betterment of mathematical education in private an public schools in Peru through a series of interrelated initiatives such as research on mathematics learning processes, editing of new textbooks based on modern learning methodologies, studies on

1. Tomado de: Delgado-Aparicio L., Álvaro; Torres Llosa, Cecilia y Ortiz de Zevallos, Gabriel. 2004. Playing with Mathematics in Peru through Internet: A case study of www.20enmate.com. Grupo Apoyo. [www.20enmate.com/pdf/EISTAcaso.pdf.

2. MINISTERIO DE EDUCACIÓN DEL PERÚ (2001). Boletín de la Unidad de Medición de la Calidad $\mathrm{N}^{\circ}$ 9. Lima. 
psychological and cognitive development, updating of teacher's teaching techniques through workshops and videos, establishing liaisons between the school system and private companies to provide poorer students with didactic materials, creating book-loan programs to ensure the sustainability of these efforts, etc.

These initiatives, channeled through Instituto Apoyo (Grupo Apoyo's non-profit branch) are part of an integrated program called Matemáticas para Todos («Mathematics for Everyone»), whose goal is to generate a structural improvement in mathematics education in Peruvian schools.

Matemáticas para Todos was born as a response to the low quality and outdated methodology of mathematics pedagogy in Peru. Instituto Apoyo developed a series of textbooks based on a new approach to learning processes, borrowed from Germany and adapted to local experiences, to renovate the quality and underlying philosophy of mathematics learning. These new textbooks were distributed nation-wide through school networks such as «Fe y Alegría» (which encompasses approximately 100 public schools) with the support of different private sector companies. Since February 2003, more than 50,000 books have been distributed in twenty-one provinces. The natural limits of printed media, the slow process of closing fundraising activities and the objective of reaching more students, however, prompted the creation of an alternative, more massive medium: www.20enmate.com, an interactive web site that expands on the Matemáticas para Todos project.

Only four months after its launching, more than 14,000 children were visiting the site regularly as registered users. The site received immediate recognition through a series of awards such as Entrepreneurial Creativity («Communications» category), IT/Users Award («Websites» category) and PC World («Best Technological Initiative with Social Awareness» category).

The following case study is partitioned as follows: first, a brief description of the www.20enmate.com website is provided; second, the development and coordination of the network that ensures the project's sustainability is described. Third, some of the project's preliminary achievements are presented. Finally, as a conclusion, some of the project's main lessons are discussed.

\section{The website}

www.20enmate.com is a free-access interactive website designed for primary and secondary school students, teachers, and parents. Interactivity stands as the key factor of this initiative, as it is what differentiates it from TV, radio or written media. As a learning process that directly involves the student, constantly demanding and reacting to his or her response, this medium is far more effective than other processes in which the student remains a passive listener or viewer. www.20enmate.com has several features that allow the student to test his or her own knowledge and performance, submit specific queries on subjects which he or she does not understand, practice through games and contests, and learn statistical data on economics, population, sports or music, thus illustrating some of the practical applications of what is being learned. 
www.20enmate.com targets students from fifth grade in primary schools to fifth grade in secondary school, (ages 9 to 16), mostly in public schools. In the first year of the pilot project, however, the focus was on students of only first and second grade of secondary schools.

The design of the page and the navigation scheme were created taking into consideration the capabilities and limitations of such an audience. Indirectly, however, the website also caters to teachers, and parents, both essential participants of the student's learning experience.

www.20enmate.com has the following sections:

- Registration: like many other web-based services, www.20enmate.com features a registration database for students to ensure traceability and build up on previous sessions. This database also generates a community of users that can be timely notified whenever a new feature or contest is launched.

- Self-Evaluation: this section allows the users to constantly test themselves in the diverse topics of their academic program, and have a clear notion of the subjects they have mastered and those they should further reinforce. As mentioned, all registered users have access to a personal database that keeps records of their performance in previous tests so they can have an accurate idea of their improvements and weaker areas. Together with the test results, the website points out the specific pages in the Matemáticas para Todos textbooks where more exercises can be found.

- Queries to Dr. Mate: this feature functions as a question drop-off box where students can freely place any query or doubt on any of the academic subjects, as well as on the use of the website. The answers are sent to the student in no longer than three days.

- Playing with Mathematics: this section reinforces mathematical knowledge and mind quickness through a variety of problem-solving games. The students with the best scores are recognized in a ranking and a series of prizes are awarded accordingly.

- The world in numbers: this section contains various statistical data sets which help the student complement and apply his or her understanding of different areas of mathematics, such as demographics, economics, music, sports or geography.

- Contests: this is a special and very popular section that periodically organizes on-line mathematics contests. Attractive prizes are offered to stimulate a healthy and tough competition.

- Teachers: this section provides teachers with pedagogic lectures and recommendations on specific chapters of the Matemáticas para Todos textbooks. The purpose of this section is to train the teachers in the new methodology and familiarize them with the characteristics and uses of the website.

- Parents: this section provides information to parents on the mathematical preparation of their children and on how to better support them in their learning processes.

The development of www.20enmate.com can be broken down into two main phases: (i) research and (ii) development. 
i) Research: in order to develop a basic conceptual draft of the product, the research process was initially focused on searching in the web for mathematics education sites. Once this research was finished, a concept draft which including a series of tentative sections was tested with several focus groups and structured in depth-interviews with different groups of children (ages 12 and 13) from both private and public schools from the Lima area. This allowed the team to gather information on the children's general Internet navigation styles as well as their impressions of the initial concept draft. The results of these focus groups provided key information that shaped the features and characteristics of the final product, and shed light on the network strategy required for its successful implementation and sustainability in time.

ii) Development. Once the concept test was finalized, an interdisciplinary team of programmers, graphic designers, system analysts, project coordinators, education specialists and teachers were organized to develop the pilot product. When the development process was finalized, another series of pilot test was conducted using additional focus groups and structured interviews.

The design and programming of www.20enmate.com used the following technological resources:

- For the data base: Microsoft SQL Server 2000

- For programming:

- Visual Interdev 6.0 (selected for its advantages in platform shows and in XML components).

- Macromedia Dreamweaver MX, which allows a better coordination between design, ASP and XML

- Visual Basic 6.0, for the design of the different components.

- Macromedia Flash MX and Action Script for the development of Animations.

- For design:

- Adobe Photoshop 7.0, for the generation of art in the site.

- Macromedia Freehand 11 for the management of vectorized images.

- Macromedia Flash MX and Adobe ImageReady 7.0 for the development of flash files and animated gifs.

- Macromedia Dreamweaver MX for the implementation of the pages.

\section{Network development}

The efforts invested in developing such a website are futile if not ably combined with an integral communications and relational strategy, capable of building a strong and efficient network of actively involved and committed participants. In this sense, the on-going process of coordinating the participation of schools, teachers, principals, Internet booth owners, volunteers, the general community, the private sector and Grupo Apoyo, is essential for the sustainability of www.20enmate.com. The partnership with Banco de Crédito del Perú, the project's main corporate sponsor, was a central instrument for the communications and diffusion operations. 
Specifically, the design and development of this network involved the following activities:

- First National On-line Mathematics Contest: launched on September 2003, this was the first contest of its kind. It tested four of the main topics of the first year secondary school curriculum (applied statistics, fractions, geometry and symmetry). The contest was widely promoted through various press conferences involving different media and key executives from both Grupo Apoyo and Banco de Crédito, as well as famous local personalities like actors and athletes. The contest lasted for a month, and generous prizes were awarded.

- Promotion in schools: alliances such as that with the Fe y Alegría school network committed school teachers and principals to promote the use of the site and their student's participation in the contests. This also involved the distribution of promotional material for the contest, together with special discount tickets in hours of service in local Internet Booths. The promotional material was also sent to other public and private schools. It is important to point out that the active involvement of teachers in this process not only increased the number of new visitors to the site, but also forced them to familiarize themselves with the Internet as a new teaching media, thus increasing their professional skills.

- Promotions in local Internet Booths: in Peru, 88\% of Internet usage takes place at Internet booths. Among children between 12 and 16 years old, $97 \%$ use Internet booths. Most notoriously, in the lower socioeconomic sector (D y E), this figure stands at $98 \% .^{3}$ In light of the importance of the network, the project demanded a specific strategy for Internet booths that would create a long-term relationship rather than one for a single promotional event. The concept of «booth partnership» was more suitable to this goal as it implied a relationship in time and a commitment to the project and its objectives. With this purpose, numerous booth owners signed a commitment act with www.20enmate.com, which accorded the use of promotion material in their business, and in exchange Apoyo covered the costs of the discount hours given to students, provided materials and merchandising as incentives, etc. The involvement of Internet booth owners was crucial as a direct source of guidance on the proper use of the website and a powerful contact for communicating future events.

- Promotions and Publicity: along with the previously described media and materials, www.20enmate.com used banners in several educational websites and sent newsletters to its registered users to promote the page and upcoming contests. Publicity was conducted through the network of agencies of Banco de Crédito del Perú and the network of clients of Grupo Apoyo.

- Student Workshops: employees from Grupo Apoyo participated in several workshops targeted to students of the Fe y Alegría school network. The objective of these workshops was to enable students to use the site's sections, take advantage of its various services, and invite them to participate in the National On-line Mathematical Contest. The employees' active involvement constituted a rich source of ideas for the improvement of

3. Source: Apoyo Opinión y Mercado. Socioeconomic sectors refer to the differences in purchasing power, where $\mathrm{A}$ is the highest and $\mathrm{E}$ lowest. 
the website and the integral project. It also served as a space to share in the sense of social responsibility and fulfillment in making a positive contribution to our country.

- Teacher Workshops: a second type of workshop was developed for teachers in order to train them in the use of the new mathematical textbooks and the website. It is important to point out that many teachers were not familiar with the use of Internet, so that such workshops not only made them better program collaborators but, also, committed them to improve their pedagogical skills.

\section{Preliminary achievements}

On September 2003, one month after the integral communication strategy was implemented and the First National Mathematics On-line Contest was launched, nearly 500,000 pages were visited and 16,000 users registered. Currently, these indicators are increasing exponentially. The project's long-term sustainability will depend on how the network is strengthened and adapted in the forthcoming years.

In terms of qualitative achievements, www.20enmate.com has accomplished the following:

- Through its system of self-evaluation, games, contests, and queries, the website has instilled a sense of autonomy and self-teaching which guarantees greater results in the long run.

- The projects strengthen the student's sense of self-esteem and personal achievement, helping him or her establish personal goals, and maintain work discipline.

- The project promotes the use of the Internet as a crucial work tool among students, teachers, and parents.

- The project breaks the prejudice that mathematics is a boring subject, exclusive to those few gifted students.

- As a powerful media, the website has also allowed more students throughout the country to learn a better mathematics curricula, promoting a more decentralized and uniform educational standard.

- Develops a sense of fair competence and personal achievement through contests, prizes and gifts.

- Promotes the interaction among students, between parents and children, and between teachers and students.

- Indirectly, and through their children, this project promotes the use of the Internet among adults, which, until then, might have had no contact with this new media.

- The project benefits Internet booth owners both financially and psychologically, as the promotional campaign not only increased the demand for their services, but also gave them the opportunity to collaborate in the betterment of their communities.

- Helped teachers improve their teaching skills, learning the uses of new technologies and methodologies for mathematics education.

- Provided volunteers and employees of Grupo Apoyo with a deeper sense of contribution to the betterment of their society. 


\section{Conclusions}

Perhaps the most important lesson learned from the www.20enmate.com experience is that the real challenge of applying the new information technologies to educational projects lies not in the design of the technologies themselves, but in the construction and perpetuation of the networks in which they operate. It is relatively easy to underestimate the organizational sophistication and the human-resource management skills required to properly administrate these complex and multi-disciplinary projects, especially one such as www.20enmate.com that aims at nothing less than a structural improvement of nation-wide mathematics education. After all, the agents involved are not only numerous -programming experts, education consultants, company directors, publicists, media, Internet booth owners, school principals, teachers, parents and children- but each one operates under different and often contrary incentives and constraints.

Finally, www.20enmate.com differs from most social responsibility projects in that it aims at a sustained and ever-growing impact on the general community, rather than focusing on a specific issue affecting the firm's immediate entourage. Only such out-reaching efforts can generate true change, the kind that translates into real competitiveness improvements and a more knowledgeable, integrated community. 